\title{
Increase in Kelch 13 Polymorphisms in Plasmodium falciparum, Southern Rwanda
}

\author{
Clara Bergmann, Welmoed van Loon, Felix Habarugira, Costanza Tacoli, Julia C. Jäger, \\ Darius Savelsberg, Fabian Nshimiyimana, Elias Rwamugema, Djibril Mbarushimana, \\ Jules Ndoli, Augustin Sendegeya, Claude Bayingana, Frank P. Mockenhaupt
}

Artemisinin resistance in Plasmodium falciparum is associated with nonsynonymous mutations in the Kelch 13 (K13) propeller domain. We found that $12.1 \%(8 / 66)$ of clinical $P$. falciparum isolates from Huye district, Rwanda, exhibited K13 mutations, including $\mathrm{R} 561 \mathrm{H}$, a validated resistance marker. $K 13$ mutations appear to be increasing in this region.

E merging artemisinin resistance to Plasmodium falciparum endangers malaria control worldwide. Currently, the resistance epicenter is the greater Mekong subregion in Southeast Asia (1). In sub-Saharan Africa, where illnesses and deaths from $P$. falciparum malaria are highest, such resistance may result in disastrous consequences (2). Early detection and close monitoring are therefore crucial.

Artemisinin resistance in P. falciparum is associated with pfkelch13 polymorphisms encoding the parasite's Kelch 13 (K13) propeller domain, which consequently serve as a molecular marker in surveillance (3). More than 200 nonsynonymous K13 single-nucleotide polymorphisms have been reported, including 11 candidate resistance mutations (i.e., associated with delayed parasite clearance) and 9 validated mutations (i.e., also reduced in vitro sensitivity) (4). Compared with those from Asia, isolates from sub-Saharan Africa show pronounced heterogeneity of nonsynonymous K13 polymorphisms, most of them rare, possibly reflecting lower drug pressure (5).

Author affiliations: Charité-Universitätsmedizin Berlin, Berlin, Germany (C. Bergmann, W. van Loon, C. Tacoli, J.C. Jäger, D. Savelsberg, F.P. Mockenhaupt); University Teaching Hospital of Butare, Butare, Rwanda (F. Habarugira, E. Rwamugema, D. Mbarushimana, J. Ndoli, A. Sendegeya); Kabutare District Hospital, Butare (F. Nshimiyimana); University of Rwanda, Kigali, Rwanda (C. Bayingana)

DOI: https://doi.org/10.3201/eid2701.203527
Rwanda achieved substantial reductions in malaria during 2006-2011, partly due to home-based management using artemether/lumefantrine (6). In 2010, at our study site in Huye district, southern Rwanda, we observed a pattern in the $P$. falciparum multidrug resistance: 1 gene suggestive of intense artemether/ lumefantrine drug pressure, whereas K13 mutations were absent. However, among P. falciparum isolates, $2.5 \%$ in 2014 and $4.5 \%$ in 2015 harbored K13 variants, including 2 candidate mutations $(7,8)$. A recent report showed the presence of a validated pfkelch13 mutation, $\mathrm{R} 561 \mathrm{H}$, at 2 sites in Rwanda (9). We conducted a cross-sectional molecular surveillance study to update records of the prevalence of $K 13$ variants in Huye among isolates collected in 2019.

\section{The Study}

During September-December 2019, we recruited study patients with uncomplicated malaria seeking treatment at the Sovu Health Centre and Kabutare District Hospital, Huye district, Rwanda. Huye district (population $\approx 390,000$ ) is located on the central plateau of Rwanda (average altitude 1,700 m, yearly rainfall $1,200 \mathrm{~mm}$, mean temperature $19^{\circ} \mathrm{C}$ ). Malaria transmission peaks in October-November and March-May. In 2010, a total of $11.7 \%$ of children had microscopically confirmed Plasmodium infection (8).

We obtained written informed consent from all participants or from the caregivers for children; we also obtained written assent from participants 7-18 years of age. The study was approved by the Rwanda National Ethics Committee. Eligibility criteria for participants included age $>1$ year; a positive result on a rapid diagnostic test, SD Bioline Malaria Ag Pf/Pan (Abbott Global Point of Care, https:/ /www.globalpointofcare. abbott); and a fever (axillary temperature $\geq 37.5^{\circ} \mathrm{C}$ ) at the time they sought treatment or within 48 hours beforehand (self-reported). We collected whole blood in S-Monovette EDTA (ethylenediaminetetraacetic 
acid; Sarstedt, https://www.sarstedt.com) tubes and confirmed malaria diagnosis by microscopy of Giemsa-stained thick blood smears; patients were also seen by a physician. We provided a 3-day regimen of artemether/lumefantrine for treatment, the first dose given under observation. All patients were asked to return after 3 days to evaluate residual parasitemia on Giemsastained thick blood smears.

Definite parasite density was counted per 200 leukocytes on Giemsa-stained thick blood smears by 2 independent microscopists, assuming a mean leukocyte count of $8,000 / \mu \mathrm{L}$. We extracted DNA using a QIAamp DNA Blood Mini kit (QIAGEN, https:/ / www.qiagen. com). Plasmodium species were typed by real-time PCRs with commercially available primers and probes for $P$. falciparum, $P$. vivax, $P$. ovale, $P$. malariae, and $P$. knowlesi (TIB MolBiol, https://www.tib-molbiol.com) on a Roche LightCycler 480 device (https:/ / lifescience. roche.com). K13 was amplified (codons $\geq 441 \leq 688$ ) by using nested PCR (3) and sequenced by a commercial provider (Eurofins Genomics, https://www.eurofinsgenomics.com). Sequences were aligned to reference K13 3D7-1343700 (PlasmoDB, https:/ / plasmodb.org) by using Geneious Prime version 2020.1 (https:// www.geneious.com). We used R version 3.6.3 (https:/ / cran.r-project.org) for statistical analysis and a binomial logistic regression model to estimate the time-trend of nonsynonymous mutations $(\mathrm{p}<0.05)$.

Of 90 patients included in the study, 74 tested positive by microscopy and PCR and 2 by PCR only. Among these patients, $51.3 \%(39 / 76)$ were female and 4 were pregnant; the median age was 18 years (range 2-69 years). $P$. falciparum was found in $88.2 \%(67 / 76)$, $P$. vivax in $7.9 \%(6 / 76), P$. ovale in $7.9 \%(6 / 76)$ and $P$. malariae in $1.3 \%(1 / 76)$. The geometric mean parasite density, based on microscopy results, was 8,926 parasites $/ \mu \mathrm{L}$ (95\% CI 5,911-13,478 parasites $/ \mu \mathrm{L}$ ); mean temperature was $37.4^{\circ} \mathrm{C}\left(\mathrm{SD} \pm 1.3^{\circ} \mathrm{C}\right)$. After 3 days of treatment, 61 malaria patients had a negative blood smear, 1 patient $(1.6 \%, 1 / 62)$ had asymptomatic parasitemia $(31,520$ parasites $/ \mu \mathrm{L})$, and 14 patients did not return for the day 3 checkup.

None of the patients infected with K13 variant parasites tested positive after 3 days of treatment. One pregnant patient sought treatment again. Initially, she had K13 wild-type parasites and was given artemether/ lumefantrine; her day 3 microscopy result was negative. Three weeks later, we detected K13 R561H parasites, possibly due to reinfection, and administered quinine.

Samples from $98 \%(66 / 67)$ of $P$. falciparum isolates were successfully sequenced for the K13 propeller domain. We found 5 different nonsynonymous polymorphisms in 8 isolates (Table); 3 harbored $\mathrm{R} 561 \mathrm{H}$, a validated resistance mutation, and the 2 candidate polymorphisms C469F and A675V (4). This finding suggests that the number of isolates with nonsynonymous K13 mutations had increased significantly over the previous decade (OR 1.4, 95\% CI 1.1-1.8; $\mathrm{p}=0.003)$.

\section{Conclusions}

Of $P$. falciparum isolates from symptomatic patients in southern Rwanda, 12\% exhibited nonsynonymous K13 mutations, a significant increase (OR 1.4, 95\% CI 1.1$1.8 ; \mathrm{p}=0.003$ ) over the previous decade compared with their absence in 2010 and 4.5\% prevalence in 2015 (7). Of note, the validated marker $\mathrm{R} 561 \mathrm{H}$ alone occurred in $4.5 \%$ of the isolates collected in 2019. Recent studies report $1 \%-3.5 \%$ of nonsynonymous K13 polymorphisms in parasite isolates from East Africa (10), whereas during 2013-2015 in Rwanda, this figure was 6.9\% (9).

The $\mathrm{R} 561 \mathrm{H}$ artemisinin resistance mutation is regularly observed across Asia (10). A recent study that reported $\mathrm{R} 561 \mathrm{H}$ in $7.4 \%$ of isolates collected during 2013-2015 in central Rwanda and 0.7\% of isolates in south-central Rwanda (9) suggested that this mutation emerged indigenously and independently from Asia $561 \mathrm{H}$ strains. We do not have data in our study to support this. None of the K13 variant parasites showed delayed clearance in our study, which may be due to the partner drug lumefantrine still being effective, similar to observations in Southeast Asia (11). In addition, the absence of delayed parasite clearance despite K13 mutations may reflect partial immunity contributing to parasite elimination (12).

We found other nonsynonymous polymorphisms only once among the isolates tested. C469F and A675V are considered artemisinin resistance candidate

\begin{tabular}{|c|c|c|c|}
\hline Year & $\begin{array}{l}\text { No. sequenced } \\
\text { isolates }\end{array}$ & $\begin{array}{c}\text { No. (\%) isolates with } \\
\text { nonsynonymous mutations }\end{array}$ & Amino acid changes and nucleotide changes \\
\hline 2010 & 75 & 0 & Not applicable \\
\hline 2014 & 81 & $2(2.5)$ & V555A, A626S \\
\hline 2015 & 66 & $3(4.5)$ & $\mathrm{P} 574 \mathrm{~L}, \dagger \mathrm{D} 648 \mathrm{H}, \mathrm{A} 675 \mathrm{~V} \dagger$ \\
\hline 2019 & 66 & $8(12.1)$ & C469F,† G533A, V555A, R561H (3×), A578S, A675V† \\
\hline
\end{tabular}

*Data during 2010-2015 derived from Tacoli et al. (7).

†Candidate mutations for artemisinin resistance.

$\ddagger$ Validated mutation for artemisinin resistance (4). 
mutations (4) and have previously been seen in East Africa $(7,13,14)$. G533A and V555A have also been previously reported in Africa but have not yet been evaluated for resistance $(7,13)$. A578S is a common K13 polymorphism across Africa but is not linked to artemisinin resistance (1).

Our study has clear limitations. Data from only 2 healthcare facilities, with limited catchment areas, were included. Adherence to treatment was assessed by patient self-report, and drug susceptibility testing was not performed. Future research should include ring-stage susceptibility assays to contribute to understanding the role of K13 mutations in Africa. Separate testing for each drug in a combination for efficacy and continued surveillance for antimicrobial resistance are needed.

Our results show that $K 13$ mutations are present in Rwanda and that their prevalence in $P$. falciparum malaria patients in the Huye District increased from $0 \%$ in 2010 to $>12 \%$ in 2019 . The validated artemisinin resistance mutation $\mathrm{R} 561 \mathrm{H}$ occurs in $4.5 \%$ of $P$. falciparum isolates being transmitted in this area. The emergence of artemisinin resistance-related mutations in Rwanda is alarming because it might indicate developing resistance against commonly used antimalarials in this region. Countermeasures need to be considered early, potentially including 3-drug antimalarial combinations (2).

\section{Acknowledgments}

We are grateful to the staff of Sovu Health Centre and Kabutare District Hospital for their collaboration and help for over 10 years.

This study was financially supported by grant GRK2046 from the German Research Foundation (DFG), which also supported W.L.C. Bergmann was supported by DFG grant GRK2290. The funding bodies had no role in designing the study, collecting, analyzing, or interpreting data, or writing the manuscript.

\section{About the Author}

Ms. Bergmann is a medical student at Charité-Universitaetsmedizin Berlin, interested in infectious disease epidemiology and tropical diseases. This manuscript forms part of her medical doctoral thesis.

\section{References}

1. Ménard D, Khim N, Beghain J, Adegnika AA, Shafiul-Alam M, Amodu O, et al. A worldwide map of Plasmodium falciparum K13-propeller polymorphisms. N Engl J Med. 2016;374:245364. https:// doi.org/10.1056/NEJMoa1513137

2. Hanboonkunupakarn B, White NJ. The threat of antimalarial drug resistance. Trop Dis Travel Med Vaccines. 2015;2:10. https://doi.org/10.1186/s40794-016-0027-8
3. Ariey F, Witkowski B, Amaratunga C, Beghain J, Langlois AC, Khim N, et al. A molecular marker of artemisinin-resistant Plasmodium falciparum malaria. Nature. 2014;505:50-5. https://doi.org/10.1038/nature12876

4. World Health Organisation Global Malaria Programme. Status report on artemisinin resistance and artemisinin-based combination therapy efficacy. Geneva: The Organisation; 2018 Aug [cited 10 May 2020]. https:/ / apps.who.int/iris/bitstream/ handle/10665/274362/WHO-CDS-GMP-2018.18-eng.pdf

5. Amato R, Miotto O, Woodrow CJ, Almagro-Garcia J, Sinha I, Campino S, et al. Genomic epidemiology of artemisinin resistant malaria. Elife. 2016;5:e08714. https://doi.org/ 10.7554/eLife.08714</unknown>

6. Uwimana A, Penkunas MJ, Nisingizwe MP, Uyizeye D, Hakizimana D, Musanabaganwa C, et al. Expanding home-based management of malaria to all age groups in Rwanda: analysis of acceptability and facility-level timeseries data. Trans R Soc Trop Med Hyg. 2018;112:513-21. https://doi.org/10.1093/trstmh/try093

7. Tacoli C, Gai PP, Bayingana C, Sifft K, Geus D, Ndoli J, et al. Artemisinin resistance-associated K13 polymorphisms of Plasmodium falciparum in southern Rwanda, 2010-2015. Am J Trop Med Hyg. 2016;95:1090-3. https:/ / doi.org/ 10.4269/ajtmh.16-0483

8. Gahutu JB, Steininger C, Shyirambere C, Zeile I, Cwinya-Ay N, Danquah I, et al. Prevalence and risk factors of malaria among children in southern highland Rwanda. Malar J. 2011;10:134. https:// doi.org/10.1186/ 1475-2875-10-134

9. Uwimana A, Legrand E, Stokes BH, Ndikumana JLM, Warsame M, Umulisa N, et al. Emergence and clonal expansion of in vitro artemisinin-resistant Plasmodium falciparum kelch13 R561H mutant parasites in Rwanda. Nat Med. 2020;26:1602-8. https:/ / doi.org/10.1038/s41591-020-1005-2

10. Ocan M, Akena D, Nsobya S, Kamya MR, Senono R, Kinengyere AA, et al. K13-propeller gene polymorphisms in Plasmodium falciparum parasite population in malaria affected countries: a systematic review of prevalence and risk factors. Malar J. 2019;18:60. https:/ / doi.org/10.1186/ s12936-019-2701-6

11. Ashley EA, Dhorda M, Fairhurst RM, Amaratunga C, Lim P, Suon S, et al. Spread of artemisinin resistance in Plasmodium falciparum malaria. N Engl J Med. 2014;371:411-23. https://doi.org/10.1056/NEJMoa1314981

12. Ataide R, Ashley EA, Powell R, Chan JA, Malloy MJ, O'Flaherty K, et al. Host immunity to Plasmodium falciparum and the assessment of emerging artemisinin resistance in a multinational cohort. Proc Natl Acad Sci U S A. 2017;114:3515-20. https://doi.org/10.1073/pnas.1615875114

13. Conrad MD, Nsobya SL, Rosenthal PJ. The diversity of the Plasmodium falciparum K13 propeller domain did not increase after implementation of artemisinin-based combination therapy in Uganda. Antimicrob Agents Chemother. 2019;63:e01234-19. https://doi.org/10.1128/AAC.01234-19

14. Ikeda M, Kaneko M, Tachibana SI, Balikagala B, Sakurai-Yatsushiro M, Yatsushiro S, et al. Artemisininresistant Plasmodium falciparum with high survival rates, Uganda, 2014-2016. Emerg Infect Dis. 2018;24:718-26. https:/ / doi.org/10.3201/eid2404.170141

Address for correspondence: Welmoed van Loon, Institute of Tropical Medicine and International Health, CharitéUniversitätsmedizin Berlin, Campus Virchow-Klinikum, Augustenburger Platz 1, 13353 Berlin, Germany; email: welmoed.vanloon@gmail.com. 